\title{
Resorbable Orthopedic Implants in Pediatric Patients: A Narrative Review
}

\author{
Phong Truong1*, Kristina Kuklova ${ }^{2}$, Gino Mercadal $^{3}$ and Diego Galindo ${ }^{4}$ \\ ${ }^{1}$ Department of Orthopedic Surgery, Larkin Community Hospital, South Miami, FL, USA \\ ${ }^{2}$ Department of General Surgery, UConn Health, Farmington, CT, USA \\ ${ }^{3}$ Lake Erie College of Osteopathic Medicine, Bradenton, FL, USA \\ ${ }^{4}$ Department of General Surgery, Sunrise Hospital and Medical Center, Las Vegas, NV, USA
}

*Corresponding author: Phong Truong, Department of Orthopedic Surgery, Larkin Community Hospital, 7000 SW 62nd Ave, Suite 401, South Miami, USA.

\begin{abstract}
The purpose of this article is to review a variety of resorbable orthopedic implants in the pediatric population. Biodegradable implants are now being widely utilized for fracture stabilization in orthopedic surgery. These materials eventually dissolve in the body, not having to perform removal operation, as is done for synthetic-based implants. The current argument in the pediatric surgical community is whether nonresorbable implants can be replaced by resorbable ones. Numbers of biodegradable polymers have been approved and safely used for fracture fixation in children. There are many potential benefits of using resorbable implants: no secondary operation for implant removal, shorter hospital stays, lessened psychological stress, and decreased risk of tissue damage have been discussed.
\end{abstract}

Abbreviations: Orthopedic; Surgery; Biodegradable; Bioabsorbable; Resorbable; Implants; Pediatrics

Abbreviations: PLLA: Poly-L-lactic acid, PLGA: Poly-lactic-co-glycolic acid, PLLA-co-PDLA: Poly-L-lactide-co-poly-DL-lactide, PHB: Polyhydroxybutyrate, CT: Computerized Tomography, MRI: Magnetic Resonance Imaging, ESIN: Elastic Stable Intramedullary Nailing, BESIN: Bioabsorbable Elastic Stable Intramedullary Nailing

\section{Introduction}

In the last 20 years, a great deal of changes have been made in the way we treat pediatric bone fractures. Children's bodies are extraordinary in their ability to heal and correct malalignments, which is why injuries are often treated nonoperatively. Lately, higher demand for implants is observed and titanium has become a conventional choice in stabilization of fractures in the pediatric population. Using rigid materials increases concerns medically, as well as economically. Secondary implant removal operations are often necessary, leading to prolonged and costly hospitalization of children. Biodegradable implants and their beneficial properties are now studied in the field of pediatric traumatology. The materials utilized become reabsorbed within the body in a matter of weeks to years, preventing kids from having unneeded procedures later on in life $[1,2]$.

\section{Discussion}

\section{What are resorbable implants}

Resorbable implants are those intended to be degraded or reabsorbed within the human body [3,4]. These biomaterials are intended to reinforce or replace organs and tissues by mimicking their functions and structural properties. In the world of orthopedic surgery, an ideal material should possess the correct yield strength, adequate biocompatibility, and homogenous degradation, in order to support and generate bone formation[1]. Another quality 
to consider is the time it takes for the implant to resorb. Some biomaterials have been shown to degrade over the course of weeks to years $[1,2]$.

The most commonly utilized implants nowadays are polymer and ceramics based. The polymer biodegradable materials are either natural or synthetic. Polymers are molecular structures composed of covalently bonded monomers. Few of the known nature-based ones are starch, chitosan, derivatives of hyaluronic acid, collagen, fibrin gels and silk [3]. The main polymer-based ones used are made up of sugar derivatives: poly-L-lactic acid (PLLA) and co-polymers poly-lactic-co-glycolic acid (PLGA) and poly-L-lactide-co-poly-DLlactide (PLLA- co-PDLA) [5,6]. Multiple reviews have shown that PLLA is the most effective of these polymers for bone implants. This is due to the similarity in the polymers reabsorption time to that required for bone repair, while still maintaining adequate physical integrity [7]. The drawback of these implants lies within its poor osteoconductivity, low strength, stiffness, and high brittleness [1,8] On the contrary, ceramics implants are defined as materials with good biocompatibility, osteoconductivity, osseointegration, and non-immunogenic properties. One article suggests that porous apatite-wollastonite glass-ceramic may be a suitable artificial bone substitute [9].

Resorbable metals, such as iron, zinc, and magnesium hold advantage over polymers and ceramics as they are more tensile, stable, and load bearing[1]. Lately, magnesium alloys have been favored in orthopedic surgery. Magnesium-based implants have demonstrated their ability to assist with bone and fracture healing[1]. It has been reported that the mechanical properties of magnesium alloys are enhanced by combining it with aluminium and rare earth elements, such as yttrium or gadolinium[3]. It aids in delaying degradation rate and increasing implant stability [1].

\section{How resorbable implants are being used}

Over the past30years, scientistshave madetremendous progress in the use of synthetic materials in the body $[10,11]$. Reabsorbable implants are now widely used in orthopedic procedures. One such example is the use of bioabsorbable screws for latarjet procedures or anterior cruciate ligament reconstruction [12-14]. Other articles have demonstrated an application of bioabsorbable screws for anterior cervical fusion and decompression in treatment of cervical spondylosis $[15,16]$. One 1996 article mentioned the use of resorbable pins for fracture fixation of small bony fragments [17]. Use of resorbable screws is safe and can prevent the complications evoked by metal implants. It may shorten the postoperative hospital stay and lessen the risk of graft extrusion and complications related to the usage of nonabsorbable devices [16].

One frequently observed indication for the use of resorbable implants is for ankle fractures. Transphyseal fixation with smalldiameter pins may be used in children, which results in financial benefits and psychological advantages of not having to remove the implants later on in life [18].
In 2013, a study mentioned new minimally invasive treatment techniques for pediatric diaphyseal forearm fractures. Ultrahigh-strength, resorbable intramedullary nails were utilized in 5-15 year old children. Patients were observed for up to one year postoperatively, and no instability, secondary movement, growth disruptions, or osteolysis was seen [1].

Another article, performed in 2015, demonstrated a novel treatment option for avascular necrosis of the femoral head and nonunion. Pure magnesium screws were combined with the vascularized iliac graft. $95 \%$ of the cases displayed hip union after an average duration of 4.1 months and avascular necrosis of the femoral head was not observed in any patient [1].

\section{Implications for pediatric orthopedic patients}

There is an ongoing debate in the pediatric surgical community on whether nonresorbable implants can be replaced by resorbable implants[1]. Titanium is commonly used for bone fracture fixation in kids. However, these implants need to be removed, having a child undergo more anesthesia and surgery. Therefore, one of the leading benefits of resorbable implants is the singular surgical procedure with definitive fixation of the fracture and minimization of anesthesia exposure [1].

One paper retrospectively studied children and adolescents treated with resorbable lactic acid polymers and trimethylene carbonate for congenital malformations, large reconstruction after tumor excision, fractures and osteotomies of the upper extremity, and hand trauma with bone and soft tissue defects. At a minimum follow up of seven months, bone healing was found to be complete. Furthermore, wound healing was uncomplicated, and foreign body reactions and infections were not observed [19].

Polymer-based implants, such as PLLA and PLGA, are widely used in children, but the degradation rate varies between weeks to years depending on the implant used. Poly-glycolic acid tends to stimulate tissue damage due to the degradation-induced changes seen with glycolic acid concentration. To counteract the implant degradation, more research should be conducted on degradation enzymes, and brittleness and low E-modulus of polymer-based implants [1].

Polyhydroxybutyrate (PHB) can act as an alternative polymer. It is another option; however, PHB has been linked to causing toxicological issues since it is produced by microorganisms. Lately, the reduction in toxicity of PHB has been observed, increasing its medical potential. And the most recent research is centered around providing proper enzymes for adequate degradation of PHB-based implants. Previously mentioned Hydroxyapatite-based ceramics implants are a conventional choice in pediatric surgery, but there are numerous disadvantages seen of the use of this material. Slow degradation rate causes the implants to stay in the human body for several years and its poor osteosynthesis properties yield a lack of load-bearing capacity. Nowadays, ceramics are still used to fill bone cysts in pediatric trauma care. 
The tensile and stable biodegradable metal, such as iron, is not an appropriate material for biodegradable metal implants, especially in children, due to its low melting point and lagging degradation rate. Zinc demonstrates many disadvantages as well: from low rigidity to corrosion inhibition, it is also not favorably seen in pediatric surgical cases. On the contrary, magnesium shows beneficial biomechanical properties, aiding in its ability to support bone fracture healing.

Recently, there has been a rise in the rate of pediatric forearm fractures, which are most commonly treated by closed reduction and casting. In the early 1980s, elastic stable intramedullary nailing (ESIN) has become a widely utilized surgical treatment of long-bone fractures in children, and in 2013 an article was written introducing a novel bioabsorbable ESIN (BESIN) technique for stabilization of diaphyseal forearm fractures in the same age group. Not having to remove the implant later on in life, has caused less psychosocial stress and morbidity caused by an additional operation [20]. Other advantages of BESIN include: no disturbance in future CT or MRI imaging post-reabsorption, avoidance of tissue retraction against metallic implants, lesser risk of tendon damage, decreased disruption of natural radius bowing during the growth of a child, and economic benefits of not having to perform a secondary implant removal procedure.

\section{Conclusion}

Utilization of resorbable implants in the pediatric population is a highly disputed topic in the field of orthopedics. Studies have observed multiple advantages of these implants compared to the nonresorbable ones in fracture care of children. No secondary operation for implant removal, shorter hospital stays, lessened psychological stress, and decreased risk of tendon and tissue damage are some of the discussed benefits. Additional research is necessary to provide insight on various types of resorbable implants in treatment of pediatric fractures.

\section{Author Contribution Statement}

Authors Phong Truong, Kristina Kuklova, Gino Mercadal and Diego Galindo contributed to the conception or design of the article; drafted or critically revised the article; approved the final version to be published; and agreed to be accountable for all aspects of the article

\section{Conflicts of Interest}

We declare that we have no conflicts of interest in the authorship or publication of this article.

\section{References}

1. Grun NG, Holweg PL, Donohue N, Klestil T, Weinberg AM (2020) Resorbable implants in pediatric fracture treatment. Innov Surg Sci 3(2): 119-125.
2. Marois $Y$, Zhang Z, Vert M, Deng X, Lenz R, et al. (2000) Mechanism and rate of degradation of polyhydroxyoctanoate films in aqueous media: A long-term in vitro study. J Biomed Mater Res 49(2): 216-224.

3. Prakasam M, Locs J, Salma-Ancane K, Loca D, Largeteau A, et al. (2017) Biodegradable materials and metallic implants-A review. J Funct Biomater 8(4): 44.

4. Nair LS, Laurencin CT (2007) Biodegradable polymers as biomaterials. Prog Polym Sci 32(8-9): 762-798.

5. Walton M, Cotton NJ (2007) Long-term in vivo degradation of poly-Llactide (PLLA) in bone. J Biomater Appl 21(4): 395-411.

6. Grizzi I, Garreau H, Li S, Vert M (1995) Hydrolytic degradation of devices based on poly (dl-lactic acid) size-dependence. Biomaterials 16(4): 305311.

7. Magarelli N, Savastano MA, Palmieri D, R Zappacosta, G Lattanzio, et al. (2007) Poly-L-lactic acid $\beta$-tricalcium phosphate screws: A preliminary in vivo biocompatibility study. Int J Immunopathol Pharmacol 20(1): 207-211.

8. Shikinami Y, Okuno M (1999) Bioresorbable devices made of forged composites of hydroxyapatite (HA) particles and poly-L-lactide (PLLA): Part I. Basic characteristics. Biomaterials 20(9): 859-877.

9. Teramoto H, Kawai A, Sugihara S, Yoshida A, Inoue H (2005) Resorption of Apatite-wollastonite containing glass-ceramic and $\beta$-tricalcium phosphate in vivo. Acta Med Okayama 59(5): 201-207.

10. Vert M (2009) Degradable and bioresorbable polymers in surgery and in pharmacology: Beliefs and facts. In: Journal of Materials Science: Materials in Medicine. 20(2): 437-446.

11. Middleton JC, Tipton AJ (2000) Synthetic biodegradable polymers as orthopedic devices. Biomaterials 21(23): 2335-2346.

12. Young AA, Maia R, Moraga C, Latif V, Liotard J-P, et al. (2010) LatarjetBristow Procedure Performed with Bioabsorbable Screws. Tech Shoulder Elb Surg 11(3): 85-89.

13. Barber FA (2005) Poly-D, L-lactide interference screws for anterior cruciate ligament reconstruction. Arthrosc - J Arthrosc Relat Surg 21(7): 804-808.

14. Schwach G, Vert M (1999) In vitro and in vivo degradation of lactic acidbased interference screws used in cruciate ligament reconstruction. In International Journal of Biological Macromolecules 25(1-3): 283-291.

15. Zhao B, Qiu X, Wang D, Li H, He X (2016) Application of bioabsorbable screw fixation for anterior cervical decompression and bone grafting. Clinics 71(6): 320-324.

16. Kim K, Isu T, Sugawara A, Matsumoto R, Isobe M (2007) Utility of new bioabsorptive screws in cervical anterior fusion. Surg Neurol 68(3):264268.

17. Claes LE, Ignatius AA, Rehm KE, Scholz C (1996) New bioresorbable pin for the reduction of small bony fragments: Design, mechanical properties and in vitro degradation. Biomaterials 17(16): 1621-1626.

18. Rokkanen PU, Bostman O, Hirvensalo E, E A Makela, E K Partio, et al. (2000) Bioabsorbable fixation in orthopaedic surgery and traumatology. Biomaterials 21(24): 2607-2613.

19. Mavrogenis AF, Kanellopoulos AD, Nomikos GN, Papagelopoulos PJ, Soucacos PN (2009) Early Experience with Biodegradable Implants in Pediatric Patients. Clin Orthop Relat Res 467(6): 1591-1598.

20. Sinikumpu J-J, Keranen J, Haltia A-M, Serlo W, Merikanto J (2013) A new mini-invasive technique in treating pediatric diaphyseal forearm fractures by bioabsorbable elastic stable intramedullary nailing: a preliminary technical report. Scand J Surg 102(4): 258-264. 